\title{
Seaweed Extract and Indoleacetic Acid Foliar Application in Relation to The Growth Performance of Sweet Pepper Grown Under Net House Conditions
}

\author{
Shahen, S. G ${ }^{1}$., A. I. A. Abido ${ }^{1}$, A. A. Alkharpotly ${ }^{2}$, F. I. Radwan ${ }^{1}$ and \\ Mona M. Yousry1. \\ ${ }^{1}$ Fac. Agric. (Saba Bash), Alex. Univ.; ${ }^{2}$ Fac. Agric. \& Nat. Resour., Aswan Univ.
}

\begin{abstract}
Sweet pepper plants (Capsicum annuum L.) grown worldwide in various distinct colours and shapes, and favors for consumers, especially those grown under protected houses. The attention of growers is directed toward enhancing or improving the growth, yield and quality of their fruits via safe agricultural practices using seaweed extract (SWE) as a biostimulant and plant growth regulators as the auxin IAA as safe alternatives for inorganic fertilization and their negative impacts. Therefore, two field experiments were carried out during two successive seasons of 2017 and 2018, to determine their influences on both vegetative and reproductive growth of sweet bell red pepper cv. 'Strick F1' grown under net house conditions. Treatments were consisted of two independent variables as foliar applicants such as seaweed extract (SWE) [Cytokan-S ] at four concentrations ( control, 500, 1000 and $2000 \mathrm{mg.l}^{-1}$ ) in combination with five concentrations of

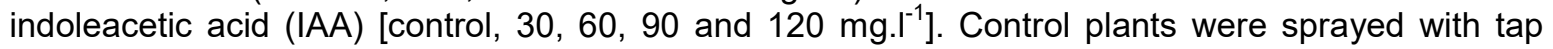
water. Pepper plants were sprayed with the assigned treatments twice, once at 15 days after transplanting, and the second one was 20 days later. Both conducted experiments were factorial experiments laid out in a randomized complete block design (RCBD), with three replicates. The obtained results showed that vegetative growth were affected significantly $(p \leq 0.05)$ due to the combination of SWE at either 1000 or $2000 \mathrm{mg} / \mathrm{l} \times$ IAA at $120 \mathrm{mg} / \mathrm{l}$; as number of leaves/plant, plant fresh and dry weights as compared to control plants. Respecting yield characters and its components, the interaction between both independent variables, exerted significant $(p \leq 0.05)$ effect on the various studied traits but without clear trends, except for fruit dry weights especially, wherein the interactions between control (SWE) $\times 120 \mathrm{mg} / \mathrm{I}(\mathrm{IAA})$, and SWE at $500 \mathrm{mg} \times \mathrm{IAA}$ at $120 \mathrm{mg} / \mathrm{l}$, gave rise to the highest average values during both seasons, in comparison to the interaction between control treatments of both variables; recorded, mostly, the least average values of the given traits. In terms of nutrient contents of fruits; N, P and K nutrient contents, the foliar application of the combination between SWE and IAA at 2000 and $120 \mathrm{mg} / \mathrm{l}$, each in turn, significantly ( $p \leq$ 0.05 ); brought about the highest average values of nutrient contents of fruits compare to the other tested combinations. In brief, this investigation suggests that foliar application with combination of both SWE at $2000 \mathrm{mg} / \mathrm{I}$ and IAA at $120 \mathrm{mg} / \mathrm{I}$ twice after 15 and 20 days later of transplanting, is recommended to achieve the best vegetative and yield and quality of the given cultivar.
\end{abstract}

Keywords: Sweet red pepper, Seaweed extract, Indoleacetic acid, vegetative growth, yield.

\section{INTRODUCTION}

Nowadays and worldwide, greenhouse sweet bell pepper (Capsicum annuum L.) grows in three different colors, and shapes. Nevertheless, the attention of both professionals and growers of greenhouse- colored grown bell peppers is directed to increase their high quality all the year-around, whereas the consumers are willing to pay more for these types of greenhouse -grown bell peppers (Smither-Kopperl and Cantliffe, 2004). Satisfying these demands require increasing the fruit set and enhancing fruit quality using natural products as seaweed extract as biostimulant and/or plant growth regulators (PGRs) as auxins for these kinds of peppers that grown under greenhouse conditions. 
Yield of bell peppers has been manipulated via various techniques as mineral (inorganic) fertilization (Leskovar et al., 1989; Qawasami et al., 1999; Omar et al., 2018). Nevertheless, some authors as Qawasami et al. (1999) reported an insignificant effect of nitrogen on total dry matter content among tested treatments, despite its high-cost, and its residues' negative impacts especially $\mathrm{N}-\mathrm{NO}_{2}$ within fruit tissues, its effect on soil physical and chemical properties. Therefore, yield of sweet bell peppers could be enhanced via natural biostimulants as seaweed extract and plant growth regulators as auxins, either solely or in combination of each other with reduction of the mineral fertilization as possible (Rouphael et al., 2018).

Seaweed extracts are macroalgae classified according to their color, and widely applied for their advantages or merits as availability the coasts, plant growth promoters, ameliorating the capabilities of plants to tolerate abiotic and biotic stresses [salinity, extreme temperature, nutrient deficiencies and drought, diseases etc.] (Calvo et al., 2014; Tsukanova et al., 2017). Seaweed extracts contain a numerous additives of growth promoters as auxins and cytokinins, in addition to macro- and micronutrients and their positive effects on many crops and horticultural commodities have been documented (Leskovar et al., 1989; Qawasami et al., 1999; Omar et al., 2018).

Auxins as IAA or auxin-like substances as phenyl-3-acetic acid (PAA) are well-known to produce endogenously in many marine algae (e.g. Sumera and Cajipe, 1981), also, has the efficacy to enhance plant growth and development for most vegetable crops. Auxins at the cellular levels affect cell elongation, cell division and the formation of adventitious roots. At the reproductive stage, foliar application of auxins or auxin-like substances can change fruit size as Capsicum annuum (Mahroon and Abbas, 2015).

In view of the above introduction review, the current research was carried out to determine the influence of both seaweed extract and the auxin indoleacetic acid at the tested concentrations on both vegetative and reproductive growth of sweet bell red pepper cv. 'Strick F1' grown under net house conditions, especially yield and its components.

\section{MATERIALS AND METHODS}

Two field experiments were carried out during the summer seasons of 2017 and 2018 in a private farm, at Abou El-Matameer city, in Behiera Governorate, Egypt, under net house (shade net 25\%) to study the effect of foliar application of the various levels of both seaweed extract (SWE) and indoleacetic acid (IAA) on vegetative growth, yield, and quality of 'Strick $F_{1}$ hybrid' sweet red pepper cultivar. Before planting, random soil samples of $0-30 \mathrm{~cm}$ depth from different places of the planting field were collected and analyzed for some important chemical and physical properties as given in Table (1). 
Pepper cultivar' seeds coined as 'Strick $F_{1}$ hybrid' was used for conducting the experimentation. Seeds were sown in seedling foam trays (84 eyes) filled with a mixture of Peat Moss: Vermiculite (1:1, v/v), supplemented with $300 \mathrm{~g}$ ammonium sulphate $(20.5 \% \mathrm{~N}), 400 \mathrm{~g}$ calcium superphosphate $\left(15 \% \quad \mathrm{P}_{2} \mathrm{O}_{5}\right), 150 \mathrm{~g}$ potassium sulphate $\left(48 \% \mathrm{~K}_{2} \mathrm{O}\right), 50 \mathrm{ml}$ micronutrient solution and $50 \mathrm{~g}$ of a fungicide (Thiophenate methyle) for each $50 \mathrm{~kg}$ of the soil mixture under plastic house conditions on Febraury $10^{\text {th }}$ during both seasons of the study. Seedlings of 60 days old were transplanted in a net house at both sides of the

Table (1). Some physical and chemical properties of the experimental site during both seasons of the experimentation ( 2017 and 2018)

\begin{tabular}{|c|c|c|}
\hline \multirow{2}{*}{ Soil properties } & \multicolumn{2}{|c|}{ Season } \\
\hline & 2017 & 2018 \\
\hline \multicolumn{3}{|l|}{ Mechanical Analysis: } \\
\hline Clay $(\%)$ & 10.00 & 10.30 \\
\hline Silt (\%) & 4.00 & 3.80 \\
\hline Sand $(\%)$ & 86.00 & 85.90 \\
\hline Textural class & Sandy loam & Sandy loam \\
\hline \multicolumn{3}{|l|}{ Chemical analysis: } \\
\hline $\mathrm{pH}(1: 1$, water suspension) & 7.40 & 7.50 \\
\hline EC ( $1: 1$, water extract $),$ dSIm & 2.58 & 2.61 \\
\hline \multicolumn{3}{|c|}{ Soluble cations in $(1: 5$, water extract), meq/l } \\
\hline $\mathrm{Ca}^{++}$ & 4.14 & 4.20 \\
\hline $\mathrm{Mg}^{++}$ & 7.16 & 7.10 \\
\hline $\mathrm{K}^{+}$ & 1.28 & 1.22 \\
\hline $\mathrm{Na}^{+}$ & 12.17 & 11.80 \\
\hline \multicolumn{3}{|c|}{ Soluble anions in (1:5, water extract), meq/l } \\
\hline $\mathrm{HCO}_{3}^{-}$ & 3.60 & 3.70 \\
\hline $\mathrm{Cl}^{-}$ & 8.40 & 8.20 \\
\hline $\mathrm{SO}_{4}^{--}$ & 12.75 & 12.42 \\
\hline $\mathrm{CaCO}_{3}(\%)$ & 9.10 & 9.30 \\
\hline Available $\mathrm{N}$ (mg/kg soil) & 531.41 & 544.33 \\
\hline Available P (mg/kg soil) & 25.00 & 26.00 \\
\hline Available K (mg/kg soil) & 175.00 & 177.00 \\
\hline
\end{tabular}

The analyses were carried out at Soil and Agricultural Chemistry Departement, The Faculty of Agricultur (Saba Basha), Alexanderia University, Egypt.

Ridge on April $13^{\text {th }}$ during the both seasons at $0.3 \mathrm{~m}$ apart and $1.5 \mathrm{~m}$ width of ridge (i. e. the initial planting density was 4.44 transplants $/ \mathrm{m}^{2}$ ). The experimental plot consisted of one ridge with $7.0 \mathrm{~m}$ long and $1.5 \mathrm{~m}$ width making an area of $10.50 \mathrm{~m}^{2}$ using drip irrigation system. Both conducted experiments were factorial experiments laid out in a randomized complete block design (RCBD), with three replicates. Each replicate included 20 treatments. All determined treatments were distributed randomly within each block. Peppers are indeterminate plants, therefore, the plants have to be pruned and trained on a regular basis to ensure a balanced growth for the maximum fruit production. 
Treatments were consisted of two factors (two independent variables) as a foliar applicants, i.e.; four concentrations of seaweed extract (SWE) as control, 500,1000 and $2000 \mathrm{mg}^{-\mathrm{I}^{-1}}$ in combination with five concentrations of indoleacetic acid (IAA) as control, 30, 60, 90 and $120 \mathrm{mg}^{-\mathrm{I}^{-1}}$. Control plants were sprayed with tap water. Both conducted experiments were factorial experiments laid out in a randomized complete block design (RCBD), with three replicates. Each replicate included 20 treatments. All determined treatments were distributed randomly within each block. Seaweed extract coined as (Cytokan-S) was used in this study. It is mixed of Ascophyllum nodosum (25\%) and Fhylum phaophyta (75\%) having the following composition: organic matter $25 \%$, inorganic matter $25 \%$, alginic acid $9 \%$, amino acid 10\%, menthol 3\%, Gibberellins 0.15\%, 6-Benziladenin $0.05 \%$ and minerals $\left(\mathrm{K}_{2} \mathrm{O} 1.5 \%, \mathrm{P}_{2} \mathrm{O}_{5} 1.5 \%\right.$, N 1\%, Ca $0.30 \%$, S $1 \%$, Se $0.03 \%$, Mg $0.30 \%$, B $75 \mathrm{mg} / \mathrm{l}$, Cu $2 \mathrm{mg} / \mathrm{l}, \mathrm{Zn} 25 \mathrm{mg} / \mathrm{l}$, Fe $75 \mathrm{mg} / \mathrm{l}$ and $\mathrm{Mn} 5 \mathrm{mg} / \mathrm{l})$. All precautions and accuracy were followed during weighing, dissolving, spraying of both independent variables. Pepper plants were sprayed with the allocated or assigned treatments twice during the growing seasons, the first one at 15 days after transplanting and the second application was 20 days later. Harvesting was accomplished after 90 days of transplanting during both seasons.

\section{Experimental data collection:}

Four plants form each treatment, in each replications, were randomly selected and tagged for recording growth attributes after 90 days of transplanting as follows:

\section{Vegetative growth:}

Plant height $(\mathrm{cm})$, number of leaves per plant, number of branches per plant, plant fresh and dry weights $(\mathrm{g})$ were recorded.

\section{Fruit number and yield parameters:}

The number of fruits per plant, average fruits weight (g), and fruits dry matter according to the following formula:

Fruits dry matter $(\%)=\frac{\text { Dry weight }}{\text { Fresh weight }} \times 100$, early yield was considered as the weight of all harvested fruits during the first 40 days of harvesting per plant $(\mathrm{g})$ and total fruit yield per square meter $(\mathrm{kg})$.

\section{Fruits $\mathbf{N}, \mathrm{P}$ and $\mathrm{K}$ contents:}

Were determined in plant tissues by using $100 \mathrm{~g}$ of fresh fruits which were taken from each treatment. The samples were dried at $70^{\circ} \mathrm{C}$ until constant weight. The obtained dry matter was ground into fine powder and $0.5 \mathrm{~g}$ of the ground dried material was digested with $\mathrm{H}_{2} \mathrm{O}_{2}$ according to Evenhuis and Dewaard (1980). Total nitrogen in digested samples was determined colorimetrically according to Chapman and Pratt (1961). Total P in digested samples was determined colorimetrically as described by Singh et al. (2005). Total K in digested samples was measured using flame photometer as described by Singh et al. (2005). 


\section{Statistical Analysis:}

All obtained data of the present study were statistically analyzed according to the design used by the MSTAT-C computer software program (Bricker, 1991). The least significant difference test at 0.05 level of probability was used to compare the differences among the given means of the various treatment combinations as illustrated by Gomez and Gomez (1984).

\section{RESULTS AND DISCUSSION}

\section{Vegetative growth:}

Average values of vegetative growth presented in Table (2) show that both independent variables (i.e. seaweed extract [SWE] and the auxin Indoleacetic acid [IAA]) and their combinations exerted significant $(p \leq 0.05)$ effects on the dependent varible traits under the study).

The main effect of SWE exhibited a direct proportional relationship with the tested traits, in general, whereas as SWE concentration increased the vegetative growth increased significantly especially at $2000 \mathrm{mg} / \mathrm{l}$ compare to control treament (foliar application with tap water). The increament percentages as an average of both seasons compare to control plants were $6.82 \%, 24.88 \%, 16.82 \%, 41.92 \%$, and $42.16 \%$ for plant height $(\mathrm{cm})$, number of leaves/plant, number of branches/plant, plant fresh weight and plant dry weight, respectively. These results are in agreement with those of Demir et al. (2006) on tomato, pepper and aubergine, Abdulraheem (2009) on cucumber, Prasad et al. (2010), Spinelli et al. (2010) on strawberry. Sridhar and Rengasamy (2012) reported that when chilli pepper (Capsicum annum) grown under filed conditions when sprayed with $1.0 \%$ of seaweed liquid fertilizer (SLF) derived from the brown seaweed Sargassum wightii, the plant growth characteristics enhanced total plant height, total fresh and dry weights, number of branches and leaf area. In this concern, it well-known that seaweed extracts are enriched with natural plant hormones as cytokinins and auxins, vitamins and some major nutrients (Sridhar and Rengasamy, 2012; Thirumaran et al., 2009 a,b). These increments in vegetative-related characters could be taken place due to component of the used SWE from plant hormones i.e. cytokinins and gibberellins that practice or exert significant effects on plant growth and development through enhancing and accelerating a number of metabolic processes as cell division and enlargement (George et al., 2008), and subsequently leading to increase these characters (Demir et al., 2006; Prasad et al., 2010; Marhoon and Abbas, 2015). Furthermore, the SWE includes unlimited amounts of micro-and macronutrients which have a crucial function in activation of many enzymes and coenzymes which participate in many biological processes; leading to cell division and enlargement (Murugalakshmikumari et al., 2002; Anantharaj and Venkatesalu, 2002). 
Table (2). Average values of some vegetative growth of sweet red pepper plants cv. 'Strick $F_{1}$ ' as affected by foliar application with seaweed extract (SWE), indoleacetic acid (IAA) and their combinations during 2017 and 2018 growing seasons

\begin{tabular}{|c|c|c|c|c|c|c|c|c|c|c|c|}
\hline \multirow{2}{*}{\multicolumn{2}{|c|}{ Treatments }} & \multicolumn{2}{|c|}{ Plant height $(\mathrm{cm})$} & \multicolumn{2}{|c|}{ No. of leaves/plant } & \multicolumn{2}{|c|}{ No. branches/plant } & \multicolumn{2}{|c|}{ Plant fresh weight $(\mathrm{g})$} & \multicolumn{2}{|c|}{ Plant dry weight $(\mathrm{g})$} \\
\hline & & 2017 & 2018 & 2017 & 2018 & 2017 & 2018 & 2017 & 2018 & 2017 & 2018 \\
\hline \multicolumn{12}{|c|}{ SWE (main effect) } \\
\hline Control & & $253.60 \mathrm{c}$ & $250.87 \mathrm{c}$ & $322.60 \mathrm{~d}$ & $327.27 \mathrm{~d}$ & $53.87 \mathrm{~d}$ & $53.13 \mathrm{~d}$ & $560.99 \mathrm{~d}$ & $577.82 \mathrm{~d}$ & $112.20 \mathrm{~d}$ & $115.56 \mathrm{~d}$ \\
\hline $500 \mathrm{mg} / \mathrm{l}$ & & $259.20 \mathrm{~b}$ & $258.53 b$ & $339.87 \mathrm{c}$ & $350.00 \mathrm{c}$ & $56.13 \mathrm{c}$ & $57.40 \mathrm{c}$ & $706.20 \mathrm{c}$ & $727.38 \mathrm{c}$ & $141.24 \mathrm{c}$ & $145.48 \mathrm{c}$ \\
\hline $1000 \mathrm{mg} / \mathrm{l}$ & & $262.53 b$ & $267.00 \mathrm{a}$ & $370.00 \mathrm{~b}$ & $381.13 b$ & $58.53 b$ & $61.13 b$ & $745.16 \mathrm{~b}$ & $767.12 \mathrm{~b}$ & $149.02 \mathrm{~b}$ & $153.47 \mathrm{~b}$ \\
\hline $2000 \mathrm{mg} / \mathrm{l}$ & & $267.66 \mathrm{a}$ & $271.20 \mathrm{a}$ & $399.86 \mathrm{a}$ & $411.67 \mathrm{a}$ & $61.13 \mathrm{a}$ & $63.87 \mathrm{a}$ & $796.17 \mathrm{a}$ & $820.05 \mathrm{a}$ & $159.23 \mathrm{a}$ & $164.52 \mathrm{a}$ \\
\hline \multicolumn{12}{|c|}{ IAA (main effect) } \\
\hline Control & & $250.58 \mathrm{c}$ & $248.83 \mathrm{c}$ & $334.75 \mathrm{~d}$ & $344.67 \mathrm{~d}$ & $52.92 \mathrm{c}$ & $52.75 \mathrm{~d}$ & $638.41 \mathrm{e}$ & $657.56 \mathrm{e}$ & $127.68 \mathrm{e}$ & $131.51 \mathrm{e}$ \\
\hline $30 \mathrm{mg} / \mathrm{l}$ & & $262.17 \mathrm{~b}$ & $262.50 \mathrm{~b}$ & $340.83 \mathrm{~d}$ & $345.00 \mathrm{~d}$ & $57.83 b$ & $59.09 \mathrm{c}$ & $663.79 \mathrm{~d}$ & $683.71 \mathrm{~d}$ & $132.75 \mathrm{~d}$ & $136.89 \mathrm{~d}$ \\
\hline $60 \mathrm{mg} / \mathrm{l}$ & & $261.42 b$ & $264.58 \mathrm{ab}$ & $352.58 \mathrm{c}$ & $363.08 \mathrm{c}$ & $57.67 \mathrm{~b}$ & 59.42 bc & $698.73 \mathrm{c}$ & $719.69 \mathrm{c}$ & $139.74 \mathrm{c}$ & $144.07 \mathrm{c}$ \\
\hline $90 \mathrm{mg} / \mathrm{l}$ & & $261.92 b$ & $265.59 \mathrm{ab}$ & $371.08 \mathrm{~b}$ & $382.33 \mathrm{~b}$ & $58.33 \mathrm{~b}$ & $60.92 \mathrm{ab}$ & $735.51 \mathrm{~b}$ & $757.57 \mathrm{~b}$ & $147.10 \mathrm{~b}$ & $151.67 \mathrm{~b}$ \\
\hline $120 \mathrm{mg} / \mathrm{l}$ & & $267.67 \mathrm{a}$ & $268.00 \mathrm{a}$ & $391.17 \mathrm{a}$ & $402.50 \mathrm{a}$ & $60.34 \mathrm{a}$ & $62.25 \mathrm{a}$ & $774.22 \mathrm{a}$ & $796.94 \mathrm{a}$ & $154.84 \mathrm{a}$ & $159.65 \mathrm{a}$ \\
\hline \multicolumn{12}{|c|}{ Combinations effect } \\
\hline SWE $(\mathrm{mg} / \mathrm{l})$ & IAA $(\mathrm{mg} / \mathrm{l})$ & & & & & & & & & & \\
\hline \multirow{5}{*}{ Control } & Control & $237.33 \mathrm{j}$ & $236.00 \mathrm{i}$ & 309.33 o & 318.67 I & $50.33 \mathrm{~h}$ & $49.33 \mathrm{i}$ & $590.16 \mathrm{~m}$ & $607.86 \mathrm{~m}$ & $118.03 \mathrm{~m}$ & $121.57 \mathrm{~m}$ \\
\hline & 30 & $253.33 \mathrm{hi}$ & $255.00 \mathrm{~g}$ & $319.67 \mathrm{I}-\mathrm{n}$ & $304.67 \mathrm{~m}$ & $53.33 \mathrm{~g}$ & $54.00 \mathrm{~g}$ & $511.85 p$ & $527.21 p$ & $102.37 p$ & $105.44 p$ \\
\hline & 60 & $256.33 \mathrm{gh}$ & $253.33 \mathrm{~g}$ & 311.33 no & 320.331 & $53.67 \mathrm{fg}$ & $52.00 \mathrm{~h}$ & 538.790 & 554.950 & 107.76 o & 110.990 \\
\hline & 90 & $259.00 \mathrm{e}-\mathrm{g}$ & $255.67 \mathrm{fg}$ & $327.33 \mathrm{kl}$ & $337.33 \mathrm{j}$ & $55.33 \mathrm{de}$ & $55.33 \mathrm{~g}$ & $567.15 n$ & $584.16 n$ & $113.43 n$ & $116.83 n$ \\
\hline & 120 & 262.00 c-e & $254.33 \mathrm{~g}$ & $345.33 \mathrm{hi}$ & $355.33 \mathrm{i}$ & $56.67 \mathrm{c}$ & $55.00 \mathrm{~g}$ & $597.00 \mathrm{~m}$ & $614.91 \mathrm{~m}$ & $119.40 \mathrm{~m}$ & $122.98 \mathrm{~m}$ \\
\hline \multirow{5}{*}{500} & Control & $251.67 \mathrm{i}$ & $247.33 \mathrm{~h}$ & 325.67 k-m & $335.33 \mathrm{jk}$ & $53.00 \mathrm{~g}$ & $52.00 \mathrm{~h}$ & 621.231 & $639.86 I$ & 124.24 I & 127.97 I \\
\hline & 30 & 261.67 d-f & $260.67 \mathrm{de}$ & $317.33 \mathrm{~m}-\mathrm{o}$ & $327.00 \mathrm{kl}$ & $56.33 \mathrm{~cd}$ & $57.67 \mathrm{f}$ & $672.46 \mathrm{j}$ & $692.64 \mathrm{j}$ & $134.49 \mathrm{j}$ & $138.53 \mathrm{j}$ \\
\hline & 60 & $259.00 \mathrm{e}-\mathrm{g}$ & 259.67 d-f & $334.00 \mathrm{jk}$ & $344.00 \mathrm{j}$ & $56.33 \mathrm{~cd}$ & $57.33 \mathrm{f}$ & $707.86 \mathrm{~h}$ & $729.09 \mathrm{~h}$ & $141.57 \mathrm{~h}$ & $145.82 \mathrm{~h}$ \\
\hline & 90 & $258.00 \mathrm{fg}$ & $261.67 \mathrm{~d}$ & $351.67 \mathrm{~h}$ & $362.33 \mathrm{hi}$ & $56.33 \mathrm{~cd}$ & $59.00 \mathrm{f}$ & $745.12 \mathrm{~g}$ & $767.47 \mathrm{~g}$ & $149.02 \mathrm{~g}$ & $153.49 \mathrm{~g}$ \\
\hline & 120 & 265.67 bc & $263.33 d$ & 370.67 ef & 381.33 ef & $58.67 \mathrm{~b}$ & $61.00 \mathrm{e}$ & $784.33 \mathrm{e}$ & $807.86 \mathrm{e}$ & $156.87 \mathrm{e}$ & $161.57 \mathrm{e}$ \\
\hline \multirow{5}{*}{1000} & Control & $254.00 \mathrm{hi}$ & $254.67 \mathrm{~g}$ & 342.67 ig & $353.33 \mathrm{i}$ & $53.67 \mathrm{fg}$ & $54.33 \mathrm{~g}$ & $653.92 \mathrm{k}$ & $673.54 \mathrm{k}$ & $130.78 \mathrm{k}$ & $134.71 \mathrm{k}$ \\
\hline & 30 & $263.33 \mathrm{~cd}$ & $263.00 \mathrm{~d}$ & $348.00 \mathrm{hi}$ & $358.67 \mathrm{i}$ & $58.67 \mathrm{~b}$ & $61.00 \mathrm{e}$ & $709.93 \mathrm{~h}$ & $731.23 \mathrm{~h}$ & $141.97 \mathrm{~h}$ & $146.23 \mathrm{~h}$ \\
\hline & 60 & 261.00 d-f & $271.00 \mathrm{bc}$ & $366.67 \mathrm{fg}$ & $377.67 \mathrm{fg}$ & $58.33 b$ & $62.67 \mathrm{de}$ & $747.30 \mathrm{~g}$ & $769.72 \mathrm{~g}$ & $149.44 \mathrm{~g}$ & $153.85 \mathrm{~g}$ \\
\hline & 90 & $261.67 d-f$ & $269.00 \mathrm{c}$ & $386.00 \mathrm{~d}$ & $397.67 \mathrm{~d}$ & $59.33 \mathrm{~b}$ & $62.33 \mathrm{de}$ & $786.63 \mathrm{e}$ & $810.23 e$ & $157.31 \mathrm{e}$ & $162.03 \mathrm{e}$ \\
\hline & 120 & $272.67 \mathrm{a}$ & $277.33 \mathrm{a}$ & $406.67 \mathrm{c}$ & $418.33 \mathrm{c}$ & $62.67 \mathrm{a}$ & $65.33 \mathrm{bc}$ & $828.03 \mathrm{c}$ & $850.87 \mathrm{c}$ & $165.59 \mathrm{c}$ & $170.55 \mathrm{c}$ \\
\hline \multirow{5}{*}{2000} & Control & $259.33 \mathrm{e}-\mathrm{g}$ & $257.33 \mathrm{e}-\mathrm{g}$ & $361.33 \mathrm{~g}$ & $371.33 \mathrm{gh}$ & 54.67 ef & $55.33 \mathrm{~g}$ & $688.33 \mathrm{i}$ & $708.98 \mathrm{i}$ & $137.67 \mathrm{i}$ & $141.80 \mathrm{i}$ \\
\hline & 30 & $270.33 a$ & $271.33 \mathrm{bc}$ & $378.33 \mathrm{de}$ & $389.67 \mathrm{de}$ & $63.00 \mathrm{a}$ & $63.67 \mathrm{~cd}$ & $760.92 \mathrm{f}$ & $783.75 f$ & $152.18 \mathrm{f}$ & $157.34 \mathrm{f}$ \\
\hline & 60 & $269.33 a b$ & $274.33 a b$ & $398.33 \mathrm{c}$ & $410.33 \mathrm{c}$ & $62.33 \mathrm{a}$ & $65.67 \mathrm{~b}$ & $800.96 \mathrm{~d}$ & $824.99 \mathrm{c}$ & $160.19 d$ & $165.62 \mathrm{~d}$ \\
\hline & 90 & $269.00 a b$ & $276.00 \mathrm{a}$ & $419.33 b$ & $432.00 \mathrm{~b}$ & $62.33 \mathrm{a}$ & $67.00 \mathrm{ab}$ & $843.12 b$ & 868.42 b & $168.62 \mathrm{~b}$ & $174.34 \mathrm{~b}$ \\
\hline & 120 & $270.33 \mathrm{a}$ & $277.00 \mathrm{a}$ & $442.00 \mathrm{a}$ & $455.00 \mathrm{a}$ & $63.33 \mathrm{a}$ & $67.67 \mathrm{a}$ & $887.50 \mathrm{a}$ & $914.12 \mathrm{a}$ & $177.50 \mathrm{a}$ & $183.50 \mathrm{a}$ \\
\hline
\end{tabular}

- Values having the same alphabetical letter (s) in common, within each column, do not significantly differ, using L.S.D. test at 0.05 level of probability. 
These results are in agreement with those of Abdulraheem (2009) on cucumber plants and with Marhoon and Abbas (2015) on sweet pepper plants (Flavio F1 and California wonder). Also, the increment percentages of number of leaves, fresh and dry weights/plant are in correlation with increasing number of branches and their leaves and their capability on photosynthesis and photosynthates in the various organs of the plant, subsequently increase organ itemed (Taiz and Zeiger, 2002).

With regard to the main effect of IAA, it is clear, also, that there is a direct proportional relationship between IAA concentrations and the dependent variable characters; whereas IAA concentration increased, such significant $(p \leq 0.05)$ effect and progressive increases in the tested traits, especially upon foliar application of IAA at $120 \mathrm{mg} / \mathrm{l}$ which brought about the highest average values of tested traits compare to control plants. The increment percentages of both seasons compare to control plants were $7.00 \%, 16.82 \%, 16.00 \%, 21.23 \%$, and $21.33 \%$ for plant height (cm), number of leaves/plant, number of branches/plant, plant fresh weight (g) and plant dry weight (g), each in turn. These results are in parallel with those of Van Pelt and Popham (2002); Hajheidari et al. (2012); Kesici et al. (2013). Kaur et al. (2017) reported that foliar spraying of IAA at $200 \mu \mathrm{M}$; led to a significant increase of Capsicum 'Indri'cv. plant height compare to control plants, which recorded the lowest average value.

Notably, plants treated with SWE exhibit multiplied one-half number of leaves versus those treated with IAA (24.88 vs. 16.82), multiplied twice of both plant fresh and plant dry weights i.e. $41.92 \%$ vs. $21.23 \%$ and $42.15 \%$ vs. $21.33 \%$, respectively. This finding could be attributed to the constituents versatility of SWE as plant growth stimulator contains mineral elements major and minor nutrients, trace elements, inorganic constituents etc. which improve plant growth and development, in general (Temple et al., 1989).

In terms of the first order interaction between both independent variables on vegetative-growth characters, it exerted significant $(p \leq 0.05)$ effect on various tested traits. Generally, it is obvious that under any concentration of SWE with IAA, more or less; the maximum average values of the studied traits compare to control plants was obvious. The interaction between SWE at either 1000 or $2000 \mathrm{mg} / \mathrm{l}$ with IAA at $120 \mathrm{mg} / \mathrm{l}$, exhibited clear differences among tested treatments as number of leaves/plant, plant fresh and dry weights as compared to control plants.

\section{Yield characters and its components:}

Concerning the results of Table (3), the average values of the tested yield characters were affected significantly due to the main effects of both independent variables compare to their control treatments except fruit fresh weight, early yield/plant and early yield $/ \mathrm{m}^{2}$ in the second season only. In most studied traits, the foliar application of SWE at $2000 \mathrm{mg} / \mathrm{l}$; recorded the highest percentage values. 
Similar trend was noticed regarding the main effect of the auxin IAA, whereas fruit dry weight character was affected significantly and differed from the other treatments, especially at $120 \mathrm{mg} / \mathrm{l}$ which recorded the highest percentage values as 30.53 and $31.27 \%$ during both seasons, respectively, compare with control treatment ( 23.77 and $25.81 \%$ during both seasons).

Regarding the first order interaction between both independent variables exerted significant $(p \leq 0.05)$ effect on the various studied traits but without clear trends. The combined treatment of SWE at either 1000 or $2000 \mathrm{mg} / \mathrm{l}$ with IAA at $120 \mathrm{mg} / \mathrm{l}$, generally, recorded the highest mean values for yield characters. 
Table (3). Average values of some yield characters of sweet red pepper plants cv. 'Strick $F_{1}$ ' as affected by foliar application with seaweed extract (SWE), indoleacetic acid (IAA) and their combinations during 2017 and 2018 growing seasons

\begin{tabular}{|c|c|c|c|c|c|c|c|c|c|c|c|}
\hline \multirow{2}{*}{\multicolumn{2}{|c|}{ Treatments }} & \multicolumn{2}{|c|}{ No. of fruits/plant } & \multicolumn{2}{|c|}{ Fruit fresh weight (g) } & \multicolumn{2}{|c|}{ Fruit dry weight (\%) } & \multicolumn{2}{|c|}{ Early yield/plant (g) } & \multicolumn{2}{|c|}{ Early yield/m2 (kg) } \\
\hline & & 2017 & 2018 & 2017 & 2018 & 2017 & 2018 & 2017 & 2018 & 2017 & 2018 \\
\hline \multicolumn{12}{|c|}{ SWE (main effect) } \\
\hline \multicolumn{2}{|c|}{ Control } & $22.33 b$ & $22.60 b$ & $148.34 b$ & $149.33 a$ & $24.89 d$ & $26.76 c$ & $828.27 b$ & $831.60 a$ & $3.67 \mathrm{~b}$ & $3.68 a$ \\
\hline \multicolumn{2}{|c|}{$500 \mathrm{mg} / \mathrm{l}$} & $23.27 \mathrm{ab}$ & $23.00 \mathrm{ab}$ & $151.67 \mathrm{ab}$ & $148.67 \mathrm{a}$ & $26.71 \mathrm{c}$ & $28.93 b$ & $881.73 a$ & $839.94 a$ & $3.91 \mathrm{a}$ & $3.73 \mathrm{a}$ \\
\hline \multicolumn{2}{|c|}{$1000 \mathrm{mg} / \mathrm{l}$} & $23.73 \mathrm{a}$ & $23.47 a b$ & $152.00 a b$ & $149.00 \mathrm{a}$ & $28.16 \mathrm{~b}$ & $30.29 a$ & $901.40 \mathrm{a}$ & $872.80 \mathrm{a}$ & $4.00 \mathrm{a}$ & $3.88 \mathrm{a}$ \\
\hline \multicolumn{2}{|c|}{$2000 \mathrm{mg} / \mathrm{l}$} & $23.33 \mathrm{ab}$ & $23.93 \mathrm{a}$ & $155.33 \mathrm{a}$ & $147.33 \mathrm{a}$ & $29.92 \mathrm{a}$ & $30.24 \mathrm{a}$ & $906.20 \mathrm{a}$ & $876.00 \mathrm{a}$ & $4.02 \mathrm{a}$ & $3.89 \mathrm{a}$ \\
\hline \multicolumn{12}{|c|}{ AA (main effect) } \\
\hline \multicolumn{2}{|c|}{ Control } & $22.09 \mathrm{~b}$ & $21.75 b$ & $146.67 \mathrm{~b}$ & $152.08 \mathrm{a}$ & $23.77 \mathrm{e}$ & $25.81 \mathrm{~d}$ & $809.33 \mathrm{~b}$ & $818.17 b$ & $3.59 \mathrm{~b}$ & $3.62 \mathrm{~b}$ \\
\hline \multicolumn{2}{|c|}{$30 \mathrm{mg} / \mathrm{l}$} & $23.25 \mathrm{a}$ & $23.17 \mathrm{a}$ & $152.08 \mathrm{a}$ & $149.58 \mathrm{ab}$ & $26.20 \mathrm{~d}$ & $28.17 \mathrm{c}$ & $883.67 \mathrm{a}$ & $860.09 a b$ & $3.92 \mathrm{a}$ & $3.82 \mathrm{ab}$ \\
\hline \multicolumn{2}{|c|}{$60 \mathrm{mg} / \mathrm{l}$} & $23.50 \mathrm{a}$ & $23.92 \mathrm{a}$ & $153.75 \mathrm{a}$ & $145.00 \mathrm{~b}$ & $27.57 \mathrm{c}$ & $29.65 b$ & $903.17 \mathrm{a}$ & $860.25 a b$ & $4.01 \mathrm{a}$ & $3.82 a b$ \\
\hline \multicolumn{2}{|c|}{$90 \mathrm{mg} / \mathrm{l}$} & $23.50 \mathrm{a}$ & $23.33 a$ & $152.92 \mathrm{a}$ & $150.00 a b$ & $29.03 b$ & $30.38 b$ & $897.92 \mathrm{a}$ & $862.92 a b$ & $3.98 \mathrm{a}$ & $3.84 \mathrm{ab}$ \\
\hline \multicolumn{2}{|c|}{$120 \mathrm{mg} / \mathrm{l}$} & $23.50 \mathrm{a}$ & $24.08 \mathrm{a}$ & $153.75 \mathrm{a}$ & $146.25 \mathrm{ab}$ & $30.53 \mathrm{a}$ & $31.27 \mathrm{a}$ & $902.92 \mathrm{a}$ & $874.00 \mathrm{a}$ & $4.00 \mathrm{a}$ & $3.88 \mathrm{a}$ \\
\hline \multicolumn{12}{|c|}{ Combinations effects } \\
\hline \multirow{5}{*}{ Control } & Control & $20.67 \mathrm{e}$ & $20.67 \mathrm{~h}$ & $141.67 \mathrm{e}$ & $148.33 \mathrm{a}-\mathrm{d}$ & $21.97 \mathrm{~m}$ & 23.631 & $732.00 \mathrm{f}$ & $776.00 \mathrm{f}$ & $3.24 \mathrm{f}$ & $3.40 \mathrm{f}$ \\
\hline & 30 & $22.33 d$ & $22.33 \mathrm{e}-\mathrm{g}$ & $150.00 \mathrm{~cd}$ & $151.67 a b$ & 23.681 & $25.46 \mathrm{k}$ & $837.00 \mathrm{e}$ & 824.00 de & $3.71 \mathrm{e}$ & $3.66 \mathrm{de}$ \\
\hline & 60 & $22.67 \mathrm{~cd}$ & $23.67 \mathrm{a}-\mathrm{d}$ & $151.67 b-d$ & $146.67 \mathrm{~b}-\mathrm{e}$ & $24.92 \mathrm{jk}$ & $26.80 \mathrm{ij}$ & 858.67 c-e & 867.33 a-d & $3.81 \mathrm{c}-\mathrm{e}$ & $3.85 \mathrm{a}-\mathrm{d}$ \\
\hline & 90 & $22.67 \mathrm{~cd}$ & $23.00 \mathrm{c}-\mathrm{f}$ & $151.67 \mathrm{~b}-\mathrm{d}$ & $151.67 a b$ & $26.24 \mathrm{gh}$ & $28.21 \mathrm{gh}$ & 858.67 c-e & 849.00 a-e & $3.81 \mathrm{c}-\mathrm{d}$ & 3.77 a-e \\
\hline & 120 & $23.33 \mathrm{a}-\mathrm{d}$ & $23.33 b-e$ & $146.67 \mathrm{de}$ & $148.33 \mathrm{a}-\mathrm{d}$ & $27.62 \mathrm{e}$ & $29.70 \mathrm{~d}-\mathrm{f}$ & $855.00 \mathrm{de}$ & 841.67 b-e & $3.79 \mathrm{de}$ & $3.74 \mathrm{c}-\mathrm{e}$ \\
\hline \multirow{5}{*}{500} & Control & $22.33 \mathrm{~d}$ & $21.67 \mathrm{gh}$ & $146.67 \mathrm{de}$ & $153.33 \mathrm{a}$ & 23.131 & $25.88 \mathrm{k}$ & $815.67 \mathrm{e}$ & 807.67 e-f & $3.62 \mathrm{e}$ & 3.59 ef \\
\hline & 30 & $23.00 \mathrm{~b}-\mathrm{d}$ & $23.33 \mathrm{~b}-\mathrm{e}$ & 153.33 a-c & $148.33 \mathrm{a}-\mathrm{d}$ & 25.52 ij & $27.44 \mathrm{hi}$ & 882.00 b-d & 864.67 a-d & $3.91 \mathrm{~b}-\mathrm{d}$ & $3.84 \mathrm{a}-\mathrm{d}$ \\
\hline & 60 & $23.33 \mathrm{a}-\mathrm{d}$ & $23.00 \mathrm{c}-\mathrm{f}$ & $153.33 \mathrm{a}-\mathrm{c}$ & $148.33 \mathrm{a}-\mathrm{d}$ & $26.86 \mathrm{fg}$ & $28.89 \mathrm{fg}$ & 894.33 a-d & $829.00 \mathrm{de}$ & 3.96 a-d & $3.68 \mathrm{de}$ \\
\hline & 90 & 23.67 a-c & $22.67 \mathrm{~d}-\mathrm{g}$ & 151.67 b-d & $151.67 a b$ & $28.28 \mathrm{~d}$ & $30.41 \mathrm{c}-\mathrm{e}$ & 897.00 a-d & 836.67 c-e & 3.98 a-d & $3.72 \mathrm{c}-\mathrm{e}$ \\
\hline & 120 & $24.00 a b$ & $24.33 \mathrm{ab}$ & $153.33 \mathrm{a}-\mathrm{c}$ & $141.67 \mathrm{e}$ & 29.77 c & $32.01 \mathrm{~b}$ & $919.67 \mathrm{ab}$ & 861.67 a-d & $4.08 \mathrm{ab}$ & $3.83 \mathrm{a}-\mathrm{d}$ \\
\hline \multirow{5}{*}{1000} & Control & $22.67 \mathrm{~cd}$ & $22.00 \mathrm{fg}$ & $151.67 \mathrm{~b}-\mathrm{d}$ & $153.33 \mathrm{a}$ & $24.35 \mathrm{k}$ & $26.18 \mathrm{jk}$ & 859.33 c-e & $843.00 \mathrm{~b}-\mathrm{e}$ & $3.81 \mathrm{c}-\mathrm{e}$ & $3.74 \mathrm{~b}-\mathrm{e}$ \\
\hline & 30 & $24.33 a$ & $23.33 \mathrm{~b}-\mathrm{e}$ & $148.33 \mathrm{~cd}$ & $148.33 \mathrm{a}-\mathrm{d}$ & $26.93 \mathrm{f}$ & $28.96 \mathrm{fg}$ & 902.00 a-c & 864.67 a-d & $4.00 \mathrm{a}-\mathrm{c}$ & $3.84 a-d$ \\
\hline & 60 & $24.33 \mathrm{a}$ & $24.67 \mathrm{a}$ & $151.67 \mathrm{~b}-\mathrm{d}$ & $141.67 \mathrm{e}$ & $28.35 d$ & $30.48 \mathrm{~cd}$ & $922.67 \mathrm{ab}$ & $873.00 \mathrm{a}-\mathrm{d}$ & $4.09 a b$ & $3.88 \mathrm{a}-\mathrm{d}$ \\
\hline & 90 & $24.00 a b$ & $23.33 \mathrm{~b}-\mathrm{e}$ & 151.67 b-d & $151.67 a b$ & 29.84 c & $32.08 \mathrm{~b}$ & $909.33 a b$ & 884.33 a-c & $4.03 \mathrm{ab}$ & $3.93 \mathrm{a}-\mathrm{c}$ \\
\hline & 120 & 23. $33 \mathrm{a}-\mathrm{d}$ & $24.00 \mathrm{a}-\mathrm{c}$ & $156.67 \mathrm{ab}$ & $150.00 \mathrm{a}-\mathrm{c}$ & $31.32 \mathrm{~b}$ & $33.77 \mathrm{a}$ & $913.67 \mathrm{ab}$ & $899.00 \mathrm{a}$ & $4.05 a b$ & $3.99 \mathrm{a}$ \\
\hline \multirow{5}{*}{2000} & Control & $22.67 \mathrm{~cd}$ & $22.67 \mathrm{~d}-\mathrm{g}$ & $146.67 \mathrm{de}$ & $153.33 \mathrm{a}$ & $25.63 \mathrm{hi}$ & $27.56 \mathrm{hi}$ & $830.33 \mathrm{e}$ & 846.00 b-e & $3.68 \mathrm{e}$ & $3.76 \mathrm{~b}-\mathrm{e}$ \\
\hline & 30 & 23.33 a-d & 23.67 a-d & $156.67 a b$ & $150.00 \mathrm{a}-\mathrm{c}$ & $28.65 d$ & $30.81 \mathrm{c}$ & $913.67 \mathrm{ab}$ & 887.00 a-c & $4.05 a b$ & $3.94 \mathrm{a}-\mathrm{c}$ \\
\hline & 60 & 23.67 a-c & $24.33 a b$ & $158.33 \mathrm{a}$ & 143.33 de & $30.16 \mathrm{c}$ & $32.43 b$ & $937.00 \mathrm{a}$ & 871.67 a-d & $4.16 \mathrm{a}$ & 3.87 a-d \\
\hline & 90 & 23.67 a-c & $24.33 a b$ & $156.67 a b$ & 145.00 c-e & $31.75 b$ & $30.81 c$ & $926.67 \mathrm{ab}$ & 881.67 a-c & $4.11 \mathrm{ab}$ & $3.92 \mathrm{a}-\mathrm{c}$ \\
\hline & 120 & $23.33 \mathrm{a}-\mathrm{d}$ & $24.67 \mathrm{a}$ & $158.33 \mathrm{a}$ & $145.00 \mathrm{c}-\mathrm{e}$ & $33.42 \mathrm{a}$ & 29.60 ef & $923.33 \mathrm{ab}$ & $893.67 \mathrm{ab}$ & $4.09 a b$ & $3.97 \mathrm{ab}$ \\
\hline
\end{tabular}


Continued..

\begin{tabular}{|c|c|c|c|c|c|}
\hline \multirow{2}{*}{\multicolumn{2}{|c|}{ Treatments }} & \multicolumn{2}{|c|}{ Fruit yield/plant (kg) } & \multicolumn{2}{|c|}{ Fruit yield/m² $(\mathrm{kg})$} \\
\hline & & 2017 & 2018 & 2017 & 2018 \\
\hline \multicolumn{6}{|c|}{ SWE (main effect) } \\
\hline Control & & $3.32 \mathrm{~b}$ & $3.38 \mathrm{~b}$ & $14.72 b$ & $14.98 \mathrm{~b}$ \\
\hline $500 \mathrm{mg} / \mathrm{l}$ & & $3.53 a$ & $3.42 a b$ & $15.67 a$ & 15.16ab \\
\hline $1000 \mathrm{mg} / \mathrm{l}$ & & $3.61 \mathrm{a}$ & $3.49 \mathrm{ab}$ & $16.01 \mathrm{a}$ & $15.51 \mathrm{ab}$ \\
\hline $2000 \mathrm{mg} / \mathrm{l}$ & & $3.63 a$ & $3.53 a$ & $16.09 a$ & $15.64 a$ \\
\hline \multicolumn{6}{|c|}{ IAA (main effect) } \\
\hline Control & & $3.25 b$ & $3.31 \mathrm{~b}$ & $14.39 \mathrm{~b}$ & $14.69 \mathrm{~b}$ \\
\hline $30 \mathrm{mg} / \mathrm{l}$ & & $3.54 a$ & $3.47 \mathrm{a}$ & $15.69 a$ & $15.38 a$ \\
\hline $60 \mathrm{mg} / \mathrm{l}$ & & $3.62 a$ & $3.47 a$ & $16.04 a$ & 15.38a \\
\hline $90 \mathrm{mg} / \mathrm{l}$ & & $3.60 \mathrm{a}$ & $3.50 \mathrm{a}$ & $15.95 a$ & $15.53 a$ \\
\hline $120 \mathrm{mg} / \mathrm{l}$ & & $3.61 \mathrm{a}$ & $3.52 a$ & $16.04 a$ & $15.62 a$ \\
\hline \multicolumn{6}{|c|}{ Combinations effect } \\
\hline $\operatorname{SWE}(\mathrm{mg} / \mathrm{l})$ & IAA $(\mathrm{mg} / \mathrm{l})$ & & & & \\
\hline \multirow{5}{*}{ Control } & Control & $2.93 \mathrm{~g}$ & $3.07 \mathrm{~g}$ & $13.00 \mathrm{~g}$ & $13.61 \mathrm{~g}$ \\
\hline & 30 & $3.35 \mathrm{ef}$ & $3.39 \mathrm{ef}$ & 14.87 ef & $15.04 \mathrm{ef}$ \\
\hline & 60 & $3.44 c-f$ & $3.47 b-e$ & $15.25 c-f$ & $15.41 b-e$ \\
\hline & 90 & $3.44 c-f$ & 3.49a-e & $15.25 c-f$ & $15.48 a-e$ \\
\hline & 120 & $3.42 d-f$ & $3.46 \mathrm{~b}-\mathrm{e}$ & $15.21 d-f$ & $15.35 b-e$ \\
\hline \multirow{5}{*}{500} & Control & $3.28 f$ & $3.32 f$ & $14.54 f$ & $14.75 f$ \\
\hline & 30 & $3.53 b-e$ & $3.46 \mathrm{~b}-\mathrm{e}$ & $15.66 \mathrm{~b}-\mathrm{e}$ & $15.36 \mathrm{~b}-\mathrm{e}$ \\
\hline & 60 & $3.58 a-d$ & $3.41 d-f$ & $15.88 a-d$ & $15.13 d-f$ \\
\hline & 90 & $3.59 a-d$ & $3.44 c-f$ & $15.93 a-d$ & $15.26 c-f$ \\
\hline & 120 & $3.68 a b$ & $3.45 c-f$ & $16.33 a b$ & $15.30 c-e$ \\
\hline \multirow{5}{*}{1000} & Control & $3.44 c-f$ & 3.37ef & $15.25 c-f$ & 14.98ef \\
\hline & 30 & 3.61a-c & $3.46 b-e$ & $16.02 a-c$ & $15.36 \mathrm{~b}-\mathrm{e}$ \\
\hline & 60 & 3.69ab & 3.49a-e & 16.39ab & 15.51a-e \\
\hline & 90 & $3.64 a b$ & $3.54 a-c$ & $16.15 a b$ & $15.71 a-c$ \\
\hline & 120 & 3.66ab & $3.6 a$ & $16.22 \mathrm{ab}$ & $15.97 a$ \\
\hline \multirow{5}{*}{2000} & Control & $3.33 f$ & 3.48a-e & $14.75 f$ & 15.43a-e \\
\hline & 30 & 3.66ab & $3.55 a-c$ & $16.22 \mathrm{ab}$ & $15.75 a-c$ \\
\hline & 60 & $3.75 a$ & 3.49a-e & $16.63 a$ & 15.48a-e \\
\hline & 90 & $3.71 \mathrm{ab}$ & 3.53a-d & $16.45 a b$ & 15.66a-d \\
\hline & 120 & 3.69ab & $3.58 \mathrm{ab}$ & 16.39ab & $15.87 \mathrm{ab}$ \\
\hline
\end{tabular}

- Values having the same alphabetical letter (s) in common, within each column, do not significantlydiffer, using L.S.D. test at 0.05 level of probability.

\section{Fruits $\mathrm{N}, \mathrm{P}$ and $\mathrm{K}$ nutrient contents:}

Results postulated in Table (4) exhibit that sweet red pepper fruits cv. 'Strick $F_{1}$ contents of $\mathrm{N}, \mathrm{P}$, and $\mathrm{K}$ were affected, significantly $(p \leq 0.05)$, by the various tested treatments under investigation and their combinations. Concerning the main effect of SWE, foliar application effect on the N, P, and $\mathrm{K}$ nutrient contents of pepper fruits, generally, the given variable significantly $(p \leq 0.05)$ affected the chemical constituents under study. The given chemical content percentages reflect a direct proportional relationship as the applied SWE increased; the highest content percentages increased. Whereas, the fruits of the given cultivar, derived from plants treated with the highest SWE level $(2000 \mathrm{mg} / \mathrm{l})$ have the highest significant percentages of $\mathrm{N}, \mathrm{P}$, and $\mathrm{K}$ fruits' content during both seasons compared to control plants which recorded the least significant average 
Table (4). Percentage values of some chemical determination of sweet red pepper fruits cv. 'Strick $F_{1}$ ' as affected by foliar application with seaweed extract (SWE), indoleacetic acid (IAA) and their combinations during 2017 and 2018 growing seasons

\begin{tabular}{|c|c|c|c|c|c|c|c|}
\hline \multirow{3}{*}{\multicolumn{2}{|c|}{ Treatments }} & \multicolumn{6}{|c|}{ Nutrient contents of fruits (\% d.w.) } \\
\hline & & \multicolumn{2}{|c|}{$\mathbf{N}$} & \multicolumn{2}{|c|}{$\mathbf{P}$} & \multicolumn{2}{|c|}{$\mathbf{K}$} \\
\hline & & 2017 & 2018 & 2017 & 2018 & 2017 & 2018 \\
\hline \multicolumn{8}{|c|}{ SWE (main effect) } \\
\hline \multirow{4}{*}{\multicolumn{2}{|c|}{$\begin{array}{c}\text { Control } \\
500 \mathrm{mg} / \mathrm{l} \\
1000 \mathrm{mg} / \mathrm{l} \\
2000 \mathrm{mg} / \mathrm{l}\end{array}$}} & $1.52 \mathrm{~d}$ & $1.82 \mathrm{~d}$ & $0.32 \mathrm{~b}$ & $0.38 \mathrm{~d}$ & $1.99 \mathrm{~d}$ & $2.39 \mathrm{~d}$ \\
\hline & & $1.61 \mathrm{c}$ & $1.93 \mathrm{c}$ & $0.38 a b$ & $0.42 \mathrm{c}$ & $2.19 \mathrm{c}$ & $2.63 \mathrm{c}$ \\
\hline & & $1.69 \mathrm{~b}$ & $2.03 \mathrm{~b}$ & $0.38 a b$ & $0.46 \mathrm{~b}$ & $2.41 \mathrm{~b}$ & $2.90 \mathrm{~b}$ \\
\hline & & $1.80 \mathrm{a}$ & $2.18 \mathrm{a}$ & $0.45 \mathrm{a}$ & $0.51 \mathrm{a}$ & $2.66 \mathrm{a}$ & $3.19 \mathrm{a}$ \\
\hline \multicolumn{8}{|c|}{ IAA (main effect) } \\
\hline \multirow{5}{*}{\multicolumn{2}{|c|}{$\begin{array}{c}\text { Control } \\
30 \mathrm{mg} / \mathrm{l} \\
60 \mathrm{mg} / \mathrm{l} \\
90 \mathrm{mg} / \mathrm{l} \\
120 \mathrm{mg} / \mathrm{l}\end{array}$}} & $1.46 \mathrm{e}$ & $1.77 \mathrm{e}$ & $0.34 \mathrm{~b}$ & $0.35 \mathrm{e}$ & $1.86 \mathrm{e}$ & $2.22 \mathrm{e}$ \\
\hline & & $1.57 \mathrm{~d}$ & $1.89 \mathrm{~d}$ & $0.36 a b$ & $0.43 d$ & $2.25 \mathrm{~d}$ & $2.69 \mathrm{~d}$ \\
\hline & & $1.65 \mathrm{c}$ & $1.99 \mathrm{c}$ & $0.38 a b$ & $0.45 \mathrm{c}$ & $2.36 \mathrm{c}$ & $2.84 \mathrm{c}$ \\
\hline & & $1.74 \mathrm{~b}$ & $2.09 \mathrm{~b}$ & $0.40 a b$ & $0.48 \mathrm{~b}$ & $2.49 \mathrm{~b}$ & $2.99 \mathrm{~b}$ \\
\hline & & $1.84 \mathrm{a}$ & $2.20 \mathrm{a}$ & $0.44 \mathrm{a}$ & $0.50 \mathrm{a}$ & $2.62 \mathrm{a}$ & $3.15 \mathrm{a}$ \\
\hline \multirow{2}{*}{\multicolumn{8}{|c|}{$\begin{array}{l}\text { Combinations effects } \\
\text { SWE (mg/l) } \quad \text { IAA }(\mathrm{mg} / \mathrm{l})\end{array}$}} \\
\hline & & & & & & & \\
\hline \multirow{5}{*}{ Control } & Control & $1.37 \mathrm{I}$ & $1.62 \mathrm{j}$ & $0.29 \mathrm{~h}$ & 0.32 I & $1.71 \mathrm{I}$ & 2.051 \\
\hline & 30 & $1.44 \mathrm{kl}$ & $1.73 \mathrm{i}$ & $0.30 \mathrm{gh}$ & $0.36 \mathrm{j}$ & $1.91 \mathrm{j}$ & $2.28 \mathrm{j}$ \\
\hline & 60 & $1.52 \mathrm{i}-\mathrm{k}$ & $1.82 \mathrm{~h}$ & $0.32 \mathrm{f}-\mathrm{h}$ & $0.38 \mathrm{i}$ & $2.00 \mathrm{i}$ & $2.40 \mathrm{i}$ \\
\hline & 90 & $1.60 \mathrm{hi}$ & $1.92 \mathrm{~g}$ & 0.34 e-h & $0.40 \mathrm{~h}$ & $2.11 \mathrm{~h}$ & $2.53 \mathrm{~h}$ \\
\hline & 120 & $1.68 \mathrm{fg}$ & $2.02 \mathrm{f}$ & $0.35 \mathrm{~d}-\mathrm{h}$ & $0.42 \mathrm{~g}$ & $2.22 \mathrm{~g}$ & $2.67 \mathrm{~g}$ \\
\hline \multirow{5}{*}{500} & Control & $1.44 \mathrm{kl}$ & $1.73 \mathrm{i}$ & $0.46 \mathrm{bc}$ & $0.34 \mathrm{k}$ & $1.81 \mathrm{k}$ & $2.16 \mathrm{k}$ \\
\hline & 30 & $1.52 \mathrm{~h}-\mathrm{j}$ & $1.83 \mathrm{~h}$ & 0.34 e-h & $0.40 \mathrm{~h}$ & $2.11 \mathrm{~h}$ & $2.54 \mathrm{~h}$ \\
\hline & 60 & $1.60 \mathrm{gh}$ & $1.92 \mathrm{~g}$ & $0.35 \mathrm{~d}-\mathrm{h}$ & $0.43 \mathrm{~g}$ & $2.23 \mathrm{~g}$ & $2.67 \mathrm{~g}$ \\
\hline & 90 & $1.69 \mathrm{f}$ & $2.02 \mathrm{f}$ & $0.37 c-h$ & $0.45 \mathrm{f}$ & $2.35 \mathrm{f}$ & $2.81 \mathrm{f}$ \\
\hline & 120 & $1.78 \mathrm{de}$ & $2.13 \mathrm{de}$ & $0.39 c-g$ & $0.48 \mathrm{e}$ & $2.47 \mathrm{e}$ & $2.96 \mathrm{e}$ \\
\hline \multirow{5}{*}{1000} & Control & $1.52 \mathrm{i}-\mathrm{k}$ & $1.82 \mathrm{~h}$ & $0.30 \mathrm{gh}$ & $0.36 \mathrm{j}$ & $1.90 \mathrm{j}$ & $2.27 \mathrm{j}$ \\
\hline & 30 & $1.60 \mathrm{gh}$ & $1.92 \mathrm{~g}$ & $0.37 \mathrm{c}-\mathrm{h}$ & $0.45 \mathrm{f}$ & $2.35 \mathrm{f}$ & $2.82 \mathrm{f}$ \\
\hline & 60 & $1.68 \mathrm{fg}$ & $2.02 \mathrm{f}$ & $0.39 \mathrm{c}-\mathrm{g}$ & $0.47 \mathrm{e}$ & $2.47 \mathrm{e}$ & $2.97 \mathrm{e}$ \\
\hline & 90 & $1.77 \mathrm{de}$ & $2.13 \mathrm{de}$ & 0.41 b-f & $0.50 \mathrm{~d}$ & $2.60 \mathrm{~d}$ & $3.13 d$ \\
\hline & 120 & $1.87 \mathrm{bc}$ & $2.24 b c$ & $0.44 \mathrm{~b}-\mathrm{d}$ & $0.52 \mathrm{c}$ & $2.74 \mathrm{c}$ & $3.29 \mathrm{c}$ \\
\hline \multirow{5}{*}{2000} & Control & 1.51 jk & $1.92 \mathrm{~g}$ & $0.32 \mathrm{f}-\mathrm{h}$ & $0.38 \mathrm{i}$ & $2.00 \mathrm{i}$ & $2.40 \mathrm{i}$ \\
\hline & 30 & 1.73 ef & 2.07 ef & 0.42 b-e & $0.50 \mathrm{~d}$ & $2.61 \mathrm{~d}$ & $3.13 d$ \\
\hline & 60 & $1.81 \mathrm{~cd}$ & $2.18 \mathrm{cb}$ & $0.44 b-d$ & $0.52 \mathrm{c}$ & $2.75 \mathrm{c}$ & $3.30 \mathrm{c}$ \\
\hline & 90 & 1.91 b & $2.29 \mathrm{~b}$ & $0.49 a b$ & $0.55 \mathrm{~b}$ & $2.90 \mathrm{~b}$ & $3.47 \mathrm{~b}$ \\
\hline & 120 & $2.02 \mathrm{a}$ & $2.42 \mathrm{a}$ & $0.57 \mathrm{a}$ & $0.58 \mathrm{a}$ & $3.05 \mathrm{a}$ & $3.66 \mathrm{a}$ \\
\hline
\end{tabular}

- Values having the same alphabetical letter (s) in common, within each column, do not significantly differ, using L.S.D. test at 0.05 level of probability.

values, whereas intermediate levels; recorded intermediate and significant $(p \leq 0.05)$ average values, too. Obviously, the increment percentages (as an average of both seasons) compare to control treatment were 19.16 for $\mathrm{N}, 37.14$ for $\mathrm{P}$, and 33.56 for $\mathrm{K}$. this effect may be due to that seaweed extracts contain small amounts of natural auxins and indoles that help stimulate rooting formation and uptake and translocate water and minerals such as N, P and $\mathrm{K}$ contents. With regard to the main effect of IAA on the studied trait of nutrient content of pepper fruits, the tabulated averages disclosed similar performance as SWE was noticed 
with IAA application, whereas as IAA levels increased significantly $(p \leq 0.05)$ progressively fruits $\mathrm{N}, \mathrm{P}$ and $\mathrm{K}$ increased correspondingly. Whereas, the percentage values responded positively, during both seasons. The maximum percentage values were recorded especially upon foliar application of IAA at 120 $\mathrm{mg} / \mathrm{l}$ compare to control treatment (as an average values of both seasons).

The intermediate levels of IAA achieved intermediate significant $(p \leq 0.05)$ percentage value, too. The increment percentages of $\mathrm{N}, \mathrm{P}$ and $\mathrm{K}$ due to apply the maximum level of IAA (i.e. $120 \mathrm{mg} / \mathrm{l}$ ) compare to control treatment were 25.16 for $\mathrm{N}, 36.14$ for $\mathrm{P}$, and 41.38 for $\mathrm{K}$. Auxin are well known for their positive impact on rooting initiation and development a strong and healthy root system assists the uptake or absorb water and mineral fastly.

With respective to the interaction between both variables and their levels, the fruit chemical contents of $\mathrm{N}, \mathrm{P}, \mathrm{K}$ had affected significantly $(p \leq 0.05)$. The foliar application of SWE with $2000 \mathrm{mg} / \mathrm{l}$ plus $120 \mathrm{mg} / \mathrm{l}$ IAA; recorded, in general, the highest percentages values of the tested contents compare to other combinations.

In this context, seaweed extract and IAA levels may react synergistically at different concentration; resulting in accumulation more elemental contents of $N, P$ and $\mathrm{K}$ within fruit tissues. Seaweed extracts have high levels of natural plant hormones especially cytokinins, gibberellins and to some extent auxins and brassinosteriods (Stirk et al., 2014) which could enhance production of gutter root mass. Greater root mass provides better uptake water and minerals and facilitates their translocation; resulting in healthier, more stress-resistant plant.

\section{REFERENCES}

Abdulraheem, S. M. (2009). Effect of nitrogen fertilizer and seaweed extracts on vegetative growth and yield of cucumber. Diyala Agric. Sci. J., 1:134-145.

Anantharaj, M. and V. Venkatesalu (2002). Studies on the effect of seaweed extracts on Dolichos biflorus. Seaweed Res. Utiln, 24(1): 129-137.

Bricker, B. (1991). MSTATC: A Micro Computer Program from the Design Management and Analysis of Agronomic Research Experiments. Michigan State Univ., USA.

Calvo, P., L. Nelson and J.W. Kloepper (2014). Agricultural uses of plant biostimulants. PI. \& Soil, 383:3-41.

Chapman, H. D. and P. F. Pratt (1961). Methods of analysis for soils, plant and water. University of California-Division of Agri. Sci. Rev. California.

Demir, N., B. Dural and K. Yildirim (2006). Effect of seaweed suspensions on seed germination of tomato, pepper and aubergine. J. Biol Sci, 6(6): 11301133.

Evenhuis, B. and P. W. Dewaard (1980). Principles and practices in plant analysis. FAO Soils bull. 38 (1): 152-163.

George, E. F., M. A. Hall and G. J. De Klerk (2008). Plant growth regulators II: cytokinins, their analogues and antagonists. In Plant propagation by tissue culture (pp. 205-226). Springer, Dordrecht. 
Gomez, K. A., and A. A. Gomez (1984). "Statistical Procedures for Agricultural Research". John Wiley and Sons, Inc., New York.pp:680.

Hajheidari, A., N. Jafari, R. Naderi and M. Dashtaki (2012). Effect of 2,4-D application on quality and quantity of ornamental pepper (Capsicum annuum L.). Ann. Bio. Res., 3 (6): 2991-2993.

Kaur, S., N. Ghai and S. K. Jindal (2017). Improvement of growth characteristics and fruit set in bell pepper (Capsicum annuum L.) through IAA application. Indian J. PI. Physiol., 22 (2): 213-220.

Kesici, M., H. Gulen, S. Ergin, E. Turhan, I. P. Ahmet and N. Koksal (2013). Heat-stress tolerance of some strawberry (Fragariaxananassa) cultivars. Notulae Botanicae Horti Agrobotanici Cluj-Napoca, 41 (1): 244-249.

Leskovar, J. M., D. J. Cantliffe and N. F. Stoffellat (1989). Pepper (Capsicum annuum L.) root growth and its relation to shoot growth in response to nitrogen. J. Hort. Sci., 64(6): 711-716.

Marhoon, I. A. and M. K. Abbas (2015). Effect of foliar application of seaweed extract and amino acids on some vegetative and anatomical characters of two sweet pepper (Capsicum Annuum L.) cultivars. Intl. J. Res. Stud. Agric. Sci. (IJRSAS) 1(1): 35-44.

Murugalakshmikumari, R., V. Ramasubramanian and K. Muthuchezhian (2002). Studies on the utilization of seaweed as an organic fertilizer on the growth and some biochemical characteristics of black gram and cucumber. J. Seaweed Res. Utiliz., 24 (1): 125-128.

Omar, E. S., A. A. A. Gabal, A. A. Alkharpotly, F. I. Radwan and A. I. A. Abido (2018). Effect of mineral, organic and bio-fertilization on sweet pepper (capsicum annum L.) grown under plastic houses conditions. J. Adv. Agric. Res., 23 (3): 402-432.

Prasad, K., A. K. Das, M. D. Oza, H. Brahmbhatt, A. K. Siddhanta, R. Meena, K. E. Eswarn, M. R. Rrajyaguru and P. K. Ghosh (2010). Detection and quantification of some plant growth regulators in a seaweed-based foliar spray employing a mass spectrometric technique sans chromatographic separation. J. Agric. Food Chem., 58(8), 4594-4601.

Qawasami, W., M. J. Mohammad, H. Najim and R. Qubursi (1999). Response of bell pepper grown inside plastic houses to nitrogen fertigation. Comm. Soil Sci. \& PI. Anal., 30 (17-18): 2499-2509.

Rouphael, Y., M. Giordano, M. Cardarelli, E. Cozzolino, M. Mori, M. C. Kyriacou, P. Bonini and G. Colla (2018). Plant-and seaweed-based extracts increase yield but differentially modulate nutritional quality of greenhouse spinach through biostimulant action. Agron., 8 (126): 2- 15.

Singh, D., P. K. Chhonker and B. S. Dwivedi (2005). Manual on soil, plant and water analysis. West Ville publishing house, New Delhi: 200.

Smither-Kopperl, M. and D. J. Cantliffe (2004). Protected agriculture as a methyl bromide alternative? Current reality and future promise. Proc. Fla. State Hort. Soc., 117: 21-27.

Spinelli, F., G. Fiori, M. Noferini, M. Sprocatti and G. Costa (2010). A novel type of seaweed extract as a natural alternative to the use of iron chelates in strawberry production. Sci. Hortic., 125(3):263-269. 
Sridhar, S. and R. Rengasamy (2012). The effect of Sargassum wightii extract on the growth, biochemical composition and yield of Capsicum annum under field trial. Intl. J. Curr. Sci., 4:35-43.

Stirk, W. A., D. Tarkowská, V. Turečová, M. Strnad and J. Staden (2014). Abscisic acid, gibberellins and brassinosteroids in Kelpak®, a commercial seaweed extract made from Ecklonia maxima. J. appl. Phycol., 26(1): 561567.

Sumera, F. C. and G. J. B. Cajipe (1981). Extraction and Partial Characterization of Auxin-Like Substances from Sargassum polycystum. Agric. Bot. Mar., 24 (3): 157-164.

Taiz, L. and E. Zeiger (2002). Plant Physiology. $3^{\text {rd }}$ Edition. Sinauer Associates. Inc., publishers, Massachusetts.

Temple, W. D., A. A. Bomke, R. A. Radley and F. B. Holl (1989). Effects of kelp (Macrocystis integrifolia and Ecklonia maxima) foliar applications on bean crop growth and nitrogen nutrition under varying soil moisture regimes. PI. \& soil, 117(1): 75-83.

Thirumaran, G., M. Arumugam, R. Arumugam and P. Anantharaman (2009 a). Effect of seaweed liquid fertilizer on growth and pigment concentration of Cyamopsis tetrogonolaba (L) Taub. Amer.-Eura. J. Agron., 2 (2): 50-56.

Thirumaran, G., M. Arumugam, R. Arumugam and P. Anantharaman (2009 b). Effect of seaweed liquid fertilizer on growth and pigment concentration of Abelmoschus esculentus (1) medikus. Amer.-Eura. J. Agron., 2 (2): 57-66.

Tsukanova, K. A., J. J. Meyer and T. N. Bibikova (2017). Effect of plant growthpromoting Rhizobacteria on plant hormone homeostasis. South African J. Bot., 113: 91-102.

Van Pelt, R. and T. W. Popham (2002). Effects of three commercially available plant growth regulators and one plant growth enhancer on pepper (Capsicum annuum L.) yield and pigment content. J. Veg. Crop Prod., 8 (1): 53-61. 


\section{الملخص العربي}

\section{الرش الورقي بمستخلص الطحالب البحرية وإندول حمض الخليك وعلاقتهما بأداء النمو للقلقل الحلو النامي تحت ظروف البيوت الشبكية}

\section{شريف جمعه بسيوني شاهين' علي إبراهيم علي عبيدو' عبد الباسط عبد السميع الخربوطلي' فتحي إبراهيم رضوان' منى محمد يسري' 'كلية الزراعة (سابا باشا) - جامعة الإسكندرية- مصر

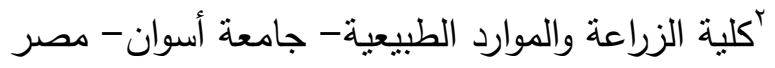

تزرع نباتات الفلفل الحلو (Capsicum annuum L) في جميع أنحاء العالم بألوان وأثنكال مختلفة، ومفضلة لدى المستهلكين خاصةً عندما تتمو تحت ظروف البيوت المظللة التي تفي بمتطلبات المستهلكين وتتعكس إيجابياً على كل من المحترفين والمزارعين. يتجه المزارعين نحو تعزيز أو تحسين نمو، إنتاجية وجودة ثمارهم من خلال الممارسات الزراعية الآمنة باستخدام عامل آمن بديل منل مستخلصات الأعثاب البحرية كمنشط حيوي طبيعي ومنظمات النمو

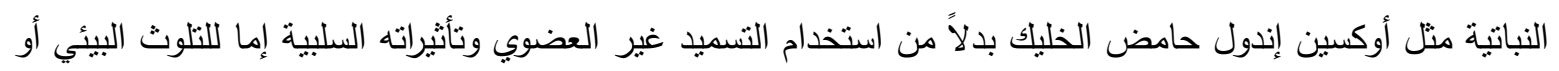

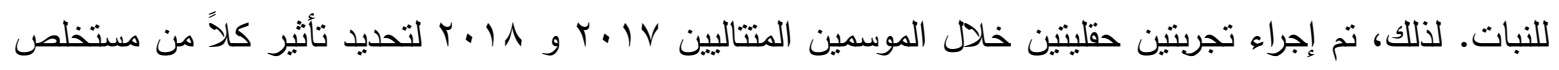
الأعثاب البحرية وأوكسين إندول حامض الخليك بتركيزات مختلفة على كلا من النمو الخضري والتكاثري لمحصول الفلفل الأحمر الحلو هجين ستريك "Strick F1" النامية تحت ظروف البيوت المظللة. وتألفت المعاملات من عاملين

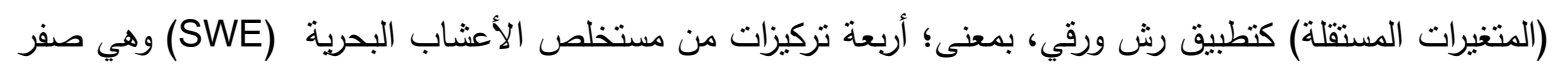

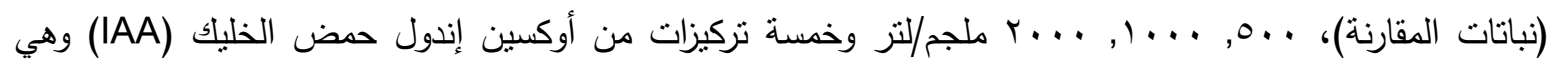

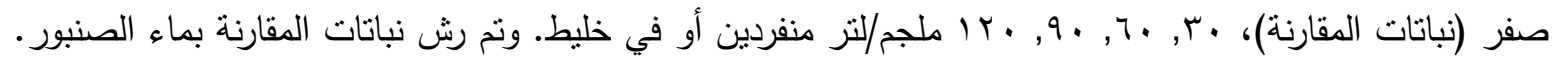

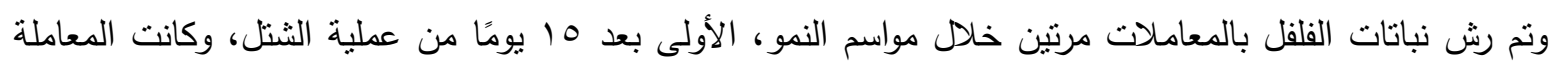
الثانية بعد • ب يومًا من الرشة الأولى. كلتا التجربتين نم تصميمهما كتجارب عاملية في تصميم القطاعات العشوائية الكاملة (RCBD) , في ثناث مكررات. أظهرت النتائج المتحصل عليها أن الرش الورقي بمستخلص الأعثاب البحرية



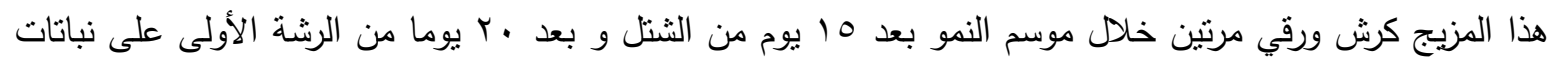
الفلفل الأحمر الحلو هجين ستريك "Strick F1" المنزرع تحت ظروف البيوت المظللة وتحت نظام الري بالتتقيط

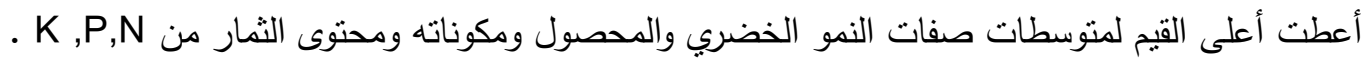


